\title{
Relationship Between EtCO2 and Quality-Parameters During Cardiopulmonary Resuscitation
}

\author{
Jesus M Ruiz ${ }^{1}$, Sofía Ruiz de Gauna ${ }^{1}$, Digna M González-Otero ${ }^{1}$, Mohamud Daya ${ }^{2}$, James K \\ Russell $^{2}$, JJ Gutiérrez ${ }^{1}$, Mikel Leturiondo ${ }^{1}$ \\ ${ }^{1}$ University of the Basque Country, Bilbao, Spain \\ ${ }^{2}$ Oregon Health \& Science University (OHSU), Portland (OR), USA
}

\begin{abstract}
Monitoring end-tidal carbon dioxide (EtCO2) is suggested as an indicator of cardiopulmonary resuscitation $(C P R)$ quality since it reflects blood flow to the lungs. However, the relationship between EtCO2 and CPR quality parameters is poorly understood. In this study, we analyzed the EtCO2 linear relation with chest compression depth, chest compression rate, and ventilation rate.

Six resuscitation episodes with the capnogram and the compression depth signal were selected to identify intervals with a stable EtCO2 value. The corresponding ventilation rate, compression depth and compression rate were annotated. Multiple linear regression was used to correlate EtCO2 changes with variations of CPR parameters, intra and inter-patient.

Median $\left(P_{25}, P_{75}\right)$ coefficient of determination $R^{2}$ per patient was $0.86(0.78,0.89)$, decreasing to 0.64 when values were considered jointly. An increase of 1 ventilation per minute caused a decrease of $1.1 \mathrm{mmHg}$ in EtCO2. An increase of $1 \mathrm{~mm}$ in depth caused an increase of $0.26 \mathrm{mmHg}$. Compression rate did not significantly influence the results.

EtCO2 reflected $C P R$ quality during resuscitation attempts, although the relationship between EtCO2 and CPR quality parameters varied between patients. The main explanatory variable was ventilation rate.
\end{abstract}

\section{Introduction}

During cardiac arrest, the quality of cardiopulmonary resuscitation (CPR) is a key determinant of outcomes. Compressions depth and rate, as well as ventilation rate are main CPR quality parameters. Current resuscitation guidelines recommend a compression depth between 5 and $6 \mathrm{~cm}$, at a rate between 100 and 120 compressions per minute, and a ventilation rate of 12 ventilations per minute for intubated patients [1]. These target values have been established on the basis of observational studies relating CPR parameters with survival [2]. Anatomic and physiological differences among patients suggest that CPR should be adjusted to optimize the hemodynamic response of each patient. Consequently, research on non-invasive indicators of blood flow during chest compressions is required. This would provide a real measurement of the CPR influence on the patient's hemodynamic response.

Capnography signal monitors end-tidal carbon dioxide concentration (EtCO2) during respiration. $\mathrm{EtCO} 2$ is a noninvasive indicator of cardiac output and pulmonary circulation $[3,4]$, and can provide a surrogate physiological measurement of the cardiac output at low flow states. Monitoring of EtCO2 during CPR was firstly proposed by Kalenda in 1978 [5]. Currently, resuscitation guidelines recommend the use of capnography by the advanced life support with intubated patients, as an indicator of CPR quality [6]. Unfortunately, quantitative relations between $\mathrm{EtCO} 2$ and CPR quality parameters are not yet well-established, and clinical studies on this topic are scarce. Two very recent observational studies have evaluated this association through the analysis of time-synchronized intervals of outof-hospital cardiac arrest resuscitation episodes [7,8].

In this study we proposed a simple EtCO2 generation model to analyze this relationship. Our hypothesis was that this model could allow establishing useful and reliable relations between EtCO2 levels and CPR quality parameters during resuscitation attempts.

\section{Materials and methods}

\subsection{Model for carbon dioxide concentra- tion in the pulmonary cavity}

Figure 1 depicts the model we proposed for the analysis of variations of carbon dioxide concentration in the pulmonary cavity. Generation of carbon dioxide in the pulmonary cavity relies on the alveolar exchange of oxygen and carbon dioxide which is proportional to pulmonary circulation. Consequently, it depends directly on the depth 
and rate of chest compressions. Ventilation is the only factor determining carbon dioxide extraction from the pulmonary cavity. Assuming equal exchanged air volume in each ventilation, extraction is exclusively governed by ventilation rate.

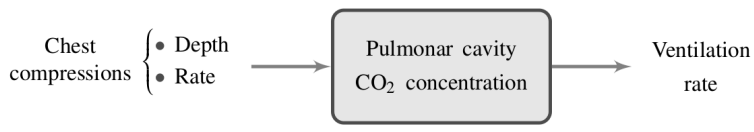

Figure 1. Proposed model for $\mathrm{CO} 2$ concentration during CPR.

A more complex model should include additional factors as previous pathologies or drugs administration during the resuscitation intervention. One key factor is that the capnogram (and therefore the EtCO2 values) is not a stationary signal. In fact, the capnogram has a noticeable inertial response to changes in the regulating parameters of the model. This behavior can be observed in Figure 2 which shows the effect of an abrupt change in compression depth on the EtCO2 level. In this example, compression depth suddenly increses from an average of $25 \mathrm{~mm}$ to $55 \mathrm{~mm}$ (at instant $980 \mathrm{~s}$, approximately), while the EtCO2 starts increasing, taking more than $1 \mathrm{~min}$ to reach an equilibrium level of $20 \mathrm{mmHg}$. This dynamic behavior of the EtCO2 level introduces a significant confusion factor that, if not properly considered, may bias the correlation results.

This study paid special attention to a careful selection of intervals during which the ETCO2 value was stationary, i.e. the value remained constant along the entire interval. Figure 3 shows an example. In this interval EtCO2 level and the rest of the parameters of the model can be considered constant.
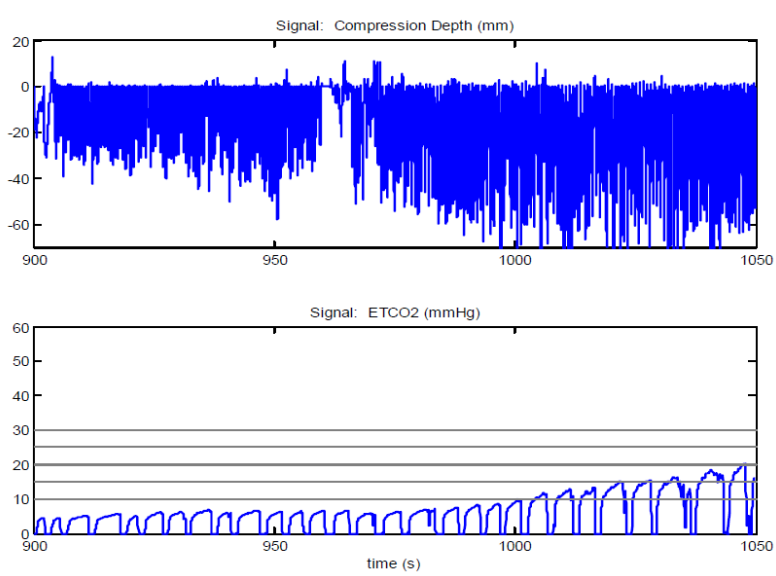

Figure 2. Example of EtCO2 increasing trend (bottom) after an abrupt change in compression depth (top).

\subsection{Data collection}

We extracted thirty episodes (one per patient) from a large out-of-hospital cardiac arrest database, compiled by the Tualatin Valley Fire \& Rescue (Tigard, OR, USA) between 2006 and 2010. Episodes were recorded by MRx monitor-defibrillators (Philips, USA). Signals of interest for our study were the capnography signal (endotracheal airway) and the compression depth signal (calculated from a chest pad, QCPR, Philips, USA). Each episode contained at least 1000 compressions. Data were provided anonymously. Capnography and compression depth signals were stored with a sampling frequency of 40 and 250 samples/s, respectively.

\subsection{Methods}

We visually reviewed the entire episodes to select those with more than eight intervals in which the EtCO2 level could be considered stationary. This selection criterion reduced our dataset drastically to six episodes. Each interval was characterized by four parameters: the EtCO2 value $(\mathrm{mmHg})$, the ventilation rate $\left(f_{v}\right.$, in ventilations per minute, vpm), both measured in the capnogram; the average compression rate $\left(f_{c}\right.$, in compressions per minute, $\mathrm{cpm})$, and the average compression depth $\left(d_{c}\right.$, in $\left.\mathrm{mm}\right)$, measured in the compression depth signal.

Figure 3 shows one of the selected intervals. Depth and rate of consecutive compressions were identified in the $t_{1-}$ $t_{2}$ interval marked in the compression depth signal (top). Average depth and rate was $44 \mathrm{~mm}$ and $89.5 \mathrm{cpm}$, respectively. Ventilation rate was calculated in the $t_{3}-t_{4}$ interval as the number of ventilations (3) divided by the interval duration $(0.575 \mathrm{~min})$. EtCO2 value was the constant value in the same interval, $15 \mathrm{mmHg}$.
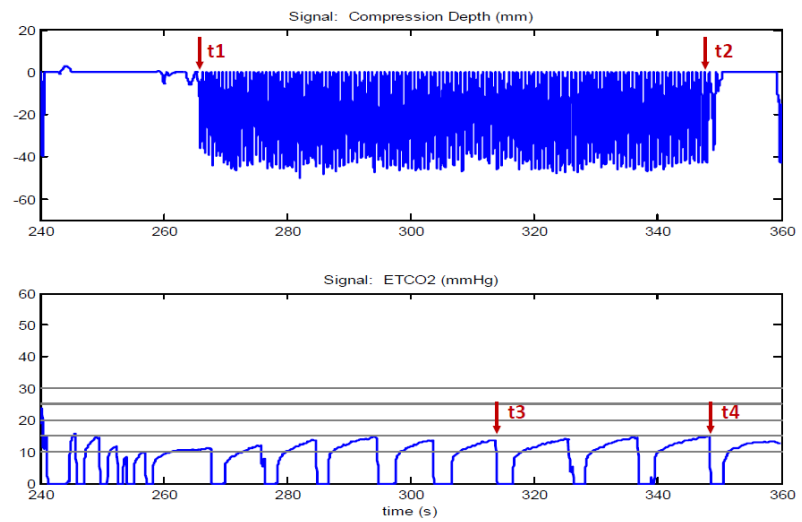

Figure 3. Stationary behavior of EtCO2 level during resuscitation. Interval in the compression depth signal (top) to obtain compression depth and rate, and in the capnogram (bottom) to obtain ventilarion rate and $\mathrm{EtCO} 2$. 


\subsection{Statistical Analysis}

Multiple linear regression was used to model the variation in $\mathrm{EtCO} 2(\triangle \mathrm{EtCO} 2)$ between consecutive intervals with respect to variations in the model parameters: $\Delta f_{v}$, $\Delta d_{c}$, and $\Delta f_{c}$. The coefficient of determination $R^{2}$ was used to assess the model goodness of fit. $R^{2}$ values were provided as median $\left(P_{25}, P_{75}\right)$, where $P_{25}$ and $P_{75}$ were the 25th and the 75th percentiles, respectively. The statistical analysis was performed both per patient and for all patients jointly.

\section{Results}

We selected a total of 63 intervals from the six episodes. A median of eleven intervals were selected per episode. Mean (SD) values per patient were 22 (8) $\mathrm{mmHg}$, 40 (4) mm, 111 (14) cpm, and 8 (2) vpm, for EtCO2 level, compression depth, compression rate, and ventilation rate, respectively.

Figure 4 shows a 3D scatter plot depicting EtCO2 variations (colored dots) with respect to variations in the model parameters for all data points.

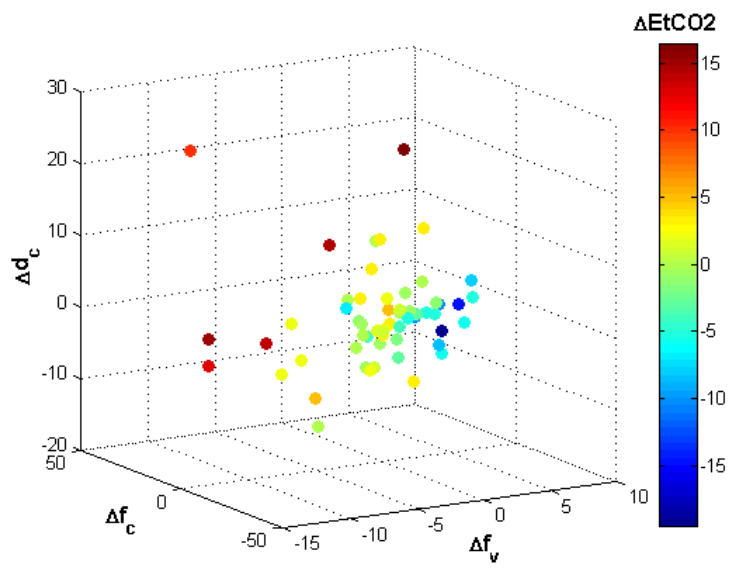

Figure 4. 3D scatter plot depicting $\triangle \mathrm{EtCO} 2$ (colored dots) with respect to $\Delta f_{v}, \Delta d_{c}$, and $\Delta f_{c}$.

Median value for $R^{2}$ was $0.86(0.78,0.89)$ in the analysis per patient, but this value decreased to 0.64 when all values were considered jointly. In both types of analysis, the main explanatory variable was $f_{v}$.

Considering the whole dataset, the regression equation was:

$$
\Delta E t C O 2=-1.09 \Delta f_{v}+0.26 \Delta d_{c}-0.02 \Delta f_{c}
$$

From the equation, it can be derived that an increase of one ventilation per minute caused a decrease of $1.1 \mathrm{mmHg}$ in the EtCO2 value. An increase of $1 \mathrm{~mm}$ in $d_{c}$ caused an increase of $0.26 \mathrm{mmHg}$. Parameter $f_{c}$ was least important, a decrease of $1 \mathrm{cpm}$ caused a decrease of $0.02 \mathrm{mmHg}$. Excluding $f_{c}$ from the model did not change $R^{2}$.

Figure 5 depicts a scatter plot showing the influence of $\Delta f_{v}$ in EtCO2 variations considering those intervals with $\Delta d_{c}<3.5 \mathrm{~mm}$. Similarly, Figure 6 shows the influence of $\Delta d_{c}$ in $\mathrm{EtCO} 2$ variations (for intervals with $\left.\Delta f_{v}<2.5 \mathrm{vpm}\right)$.

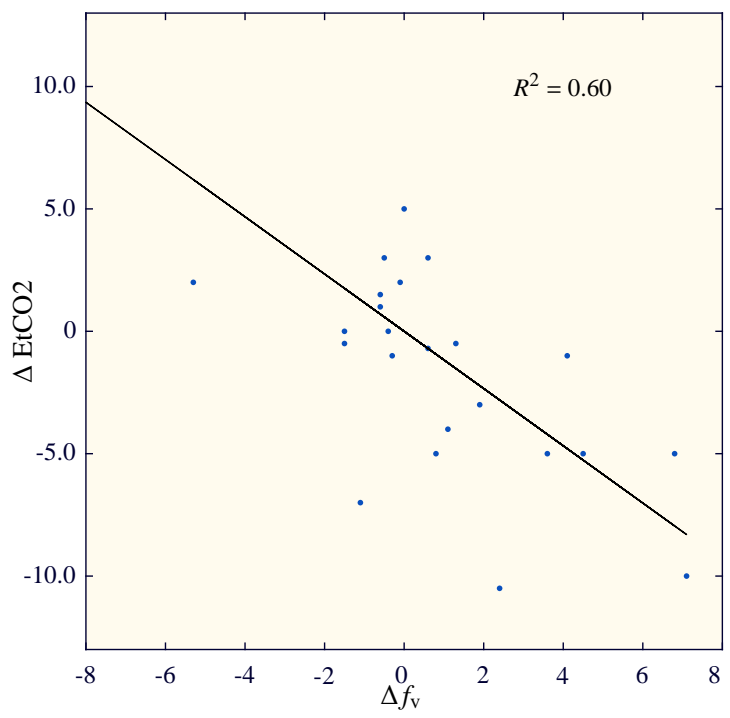

Figure 5. EtCO2 correlation with ventilation rate variations $\left(\Delta d_{c}<3.5 \mathrm{~mm}\right)$.

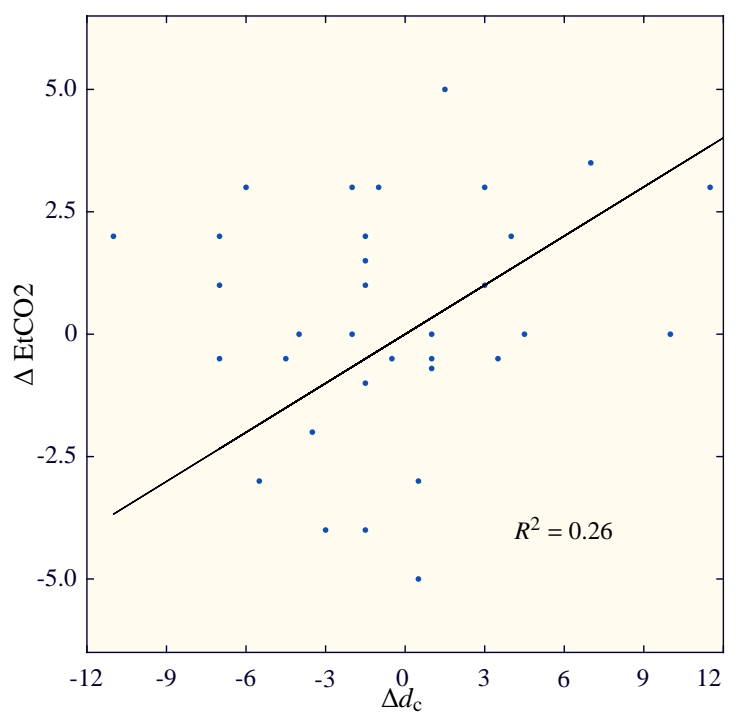

Figure 6. EtCO2 correlation with compression depth variations $\left(\Delta f_{v}<2.5 \mathrm{vpm}\right)$. 


\section{Discussion}

Chest compressions and ventilations during CPR contribute to artificially maintain a minimal flow of oxygenated blood to the vital organs. During advanced life support interventions, non-invasive capnography is proposed as a good indicator of blood flow and CPR quality.

Despite many factors influencing EtCO2 we proposed a simple model for carbon dioxide exchange being governed by three quality parameters: compression depth, compression rate, and ventilation rate. The selection of stationary EtCO2 intervals allowed the analysis of the $\mathrm{EtCO} 2$ equilibrium level reached after changes in the model factors, avoiding bias due to EtCO2 dynamics.

Our results showed a noticeable correlation between EtCO2 and quality parameters $\left(R^{2}=0.64\right)$, which increased when patients were analyzed separately $\left(R^{2}=\right.$ 0.86 ). The main explanatory variable was ventilation rate, which showed an inverse correlation with EtCO2 level. Compression depth showed a much lower but direct correlation, and compression rate barely influenced $\mathrm{EtCO} 2$ variations.

These observed trends were aligned with two recent studies with a similar objective $[7,8]$. Ventilation rate was the main factor in all studies: an increase of 10 ventilation per minute caused a decrease in $\mathrm{EtCO} 2$ of $3.0 \mathrm{mmHg}$ [7], of $16.8 \%$ [8], and of $11.0 \mathrm{mmHg}$ in our study. Compression depth was a significant predictor of increased EtCO2: an increase of $10 \mathrm{~mm}$ in depth caused an increase of $1.4 \mathrm{mmHg}$ [7], of $4.0 \%$ [8], and of $2.6 \mathrm{mmHg}$ in our study.

Differences could be explained by the selection of the time-synchronized intervals. Duration was $15 \mathrm{~s}$ in [7] $1 \mathrm{~min}$ in [8], and variable in our study. The slow response of EtCO2 level to variations in its determining factors could introduce a confusion factor in the analysis if non-stationary EtCO2 intervals are included. We think separated analysis of stationary and non-stationary behavior of EtCO2 could provide valuable information to better understand and explain its relation with CPR quality parameters. Nevertheless, we should conduct studies with larger databases to ensure generalization of the results.

\section{Conclusion}

In our study, variations in EtCO2 correlated with variations in ventilation rate and in compression depth during CPR, although this relationship varied between patients. The main explanatory variable was ventilation rate. Our results were aligned with those reported in larger observational studies, although dynamics of $\mathrm{EtCO} 2$ should be considered carefully in order to assess this relationship.

\section{Acknowledgements}

This work received financial support from the Spanish Government through the project TEC2012-31144, and through the Vice Chancellor's office for Research of the University of the Basque Country, UPV/EHU, through the grant Contratación de doctores recientes hasta su integración en programas de formación postdoctoral.

\section{References}

[1] Kleinman ME, Brennan EE, Goldberger ZD, Swor RA, Terry M, Bobrow BJ, Gazmuri RJ, Travers AH, Rea T. Part 5: Adult basic life support and cardiopulmonary resuscitation quality 2015 american heart association guidelines update for cardiopulmonary resuscitation and emergency cardiovascular care. Circulation 2015;132(18 suppl 2):S414-S435.

[2] Travers AH, Perkins GD, Berg RA, Castren M, Considine J, Escalante R, Gazmuri RJ, Koster RW, Lim SH, Nation KJ, et al. Part 3: Adult basic life support and automated external defibrillation 2015 international consensus on cardiopulmonary resuscitation and emergency cardiovascular care science with treatment recommendations. Circulation 2015; 132(16 suppl 1):S51-S83.

[3] Jin X, Weil MH, Tang W, Povoas H, Pernat A, Xie J, Bisera J. End-tidal carbon dioxide as a noninvasive indicator of cardiac index during circulatory shock. Critical care medicine 2000;28(7):2415-2419.

[4] Isserles SA, Breen PH. Can changes in end-tidal pco2 measure changes in cardiac output?. Anesthesia Analgesia 1991; 73(6):808-814.

[5] Kalenda Z. The capnogram as a guide to the efficacy of cardiac massage. Resuscitation 1978;6(4):259-263.

[6] Link MS, Berkow LC, Kudenchuk PJ, Halperin HR, Hess EP, Moitra VK, Neumar RW, O?Neil BJ, Paxton JH, Silvers SM, et al. Part 7: adult advanced cardiovascular life support 2015 american heart association guidelines update for cardiopulmonary resuscitation and emergency cardiovascular care. Circulation 2015;132(18 suppl 2):S444-S464.

[7] Sheak KR, Wiebe DJ, Leary M, Babaeizadeh S, Yuen TC, Zive D, Owens PC, Edelson DP, Daya MR, Idris AH, et al. Quantitative relationship between end-tidal carbon dioxide and cpr quality during both in-hospital and out-of-hospital cardiac arrest. Resuscitation 2015;89:149-154.

[8] Murphy RA, Bobrow BJ, Spaite DW, Hu C, McDannold R, Vadeboncoeur TF. Association between prehospital cpr quality and end-tidal carbon dioxide levels in out-of-hospital cardiac arrest. Prehospital Emergency Care 2016;20(3):369_ 377.

Address for correspondence:

Sofia Ruiz de Gauna

Department of Communications Engineering

Faculty of Engineering

Alameda Urquijo s/n, 48013-Bilbao (Spain) 